\title{
Particularités biologiques de la souche ouest-africaine de Trichinella spiralis (Owen, 1835) Réceptivité et sensibilité de quelques mammifères domestiques et sauvages
}

\author{
par S. GRÉTILLAT ef G. VASSILIADĖS
}

\begin{abstract}
RÉSUMÉ
Plusieurs séries de tests de réceptivité et de sensibilité réalisés sur 23 chats, 6 chiens, 14 porcs, 22 rats, 30 souris, 17 rats de Gambie (Cricetomys gambionus), 17 lapins, 9 hérissons (Afelerix albiventris) et 3 singes, infestés expérimentalement avec la souche ouest-africaıne de -Trichinella spiralis démontrent :

a) Les très grandes réceptivité et sensibilité du chat domestique pour lequel elle est moyennement pathogène. Les troubles marbıdes sont limités à une diarrhée hémorragique devenant chronıque dans les cas d'infestation massive Le taux de parasitisme musculaire por larves enkystées est pratıquement proportionnel à lo dose infestante adminıstrée (nombre de kystes infestants ıngérés par 100 grammes de poids vif d'hôte).

b) Le chien, moins bon hôte que le chat est cependant très sensıble. Les résultats trouvés aux autopsies de contrôle ne sont pas proportionnels aux doses infestantes.

c) Pour le porc domestique (race européenne Large White) une très faible sensibilité. Une « barrière intestinale » détruit la majorité des larves infestantes avant leur transformation en adultes et les quelques larves migratrices qui parviennent au niveau des muscles subissent un processus de calcification dès leur enkystement ou dans les quelques jours ou quelques semaines suivantes. Cependant, chez un certain nombre d'individus, quelques kystes survivent ef demeurent infestants pendant plusieurs mois, ce qui n'exclue pas le danger d'apparition de cas de frıchınose porcine par passages successifs sur le même hôte.

d) Les rongeurs sauvages, le rat, la souris et le rat de Gambie sont de très mauvais hôtes. Sujets réfractaires, enkystement anormal des larves, absence d'enkystement, calcification des kystes et des larves avec stérilisatıon de l'organisme queiques semaines après l'infestalion.

En conséquence, les rongeurs sauvages peuvent difficilement jouer un rôle dans l'épidémiologıe et la dispersıon de la trichınose en Afrique de l'Ouest.

e) Le hérisson local (Atelerix albiventris) ne joue aucun rôle dans cette épidémiologie.

f) Le lapin, en tant qu'hôte expérimental de laboratoıre, réagit très mal vis-à-vis de cefte souche et sa réceptivité est trop incerlaine et variable pour le
\end{abstract}


considérer comme un matériel susceptible d'être utilisé à la conservation de la souche.

g) Les Primates (singes Papio popio et Erythrocebus patas) sont extrêmement sensibles. Une dose de quelques centaines de kystes est mortelle pour un jeune cynocéphale.

Ce sont donc les carnivores et particulièrement les Félidés qui sont les hôtes de choix pour la trichinose en Afrique de l'Ouest. Les réservorrs de parasites étant les carnivores sauvages, le phacochère n'étant qu'un hôte accidentel (6 à 10 p. 100 des animaux abattus examinés), qui s'infeste difficilement tout comme le porc domestique.

Isolée en janvier et février 1967 à partir de trois phacochères (Phacochoerus aethiopicus) et d'un chacal (Canis adustus) abattus dans la région du Delta du Fleuve Sénégal, une souche de Trichinellaspiralis (OWEN, 1835) estentretenue sur chat au Laboratore national de l'Elevage et de Recherches vétérinaires de Dakar (Séné$\mathrm{gal}$ ).

Dès les premiers passages (essais d'infestation expérimentale faits sur chats, chiens, rats blancs, rats noirs et souris) les carnivores domestiques se révèlent très sensibles alors que le rat et la souris sont très peu réceptifs (GRÉTILLAT ef VASSILIADĖS, 1967).

Des caractères biologiques à peu près semblables ont été signalés pour la souche de T. spiralis du Kenya (NELSON et MUKUNDI, 1963 ; NELSON et BLACKIE, 1966 ; KOZAR et KOZAR, 1965).

L'examen morphologique des adultes mâles et femelles obtenus expérimentalement sur chat et sur chien ne révèle aucune différence avec l'espèce classique. Les caractères particuliers observés au Laboratoire au sujet de la réceptivité et de la sensibılité de quelques mammifères domestiques et sauvages sont donc essentiellement d'ordre biologique.

La trichinose des animaux sauvages en Afrique doit être considérée comme un danger latent.

Devant les risques de dispersion de cette zoonose qui peut avoir de graves répercussions au point de vue sanitaire et économique, des essais d'ınfestation artificielle ont été réalisés au laborataire sur chat, chien, porc, rat blanc, souris iblanche, rat de Gambie (Cricetomys gambianus), hérisson (Atelerix albiventris), lapin et singe.

Pour chacune de ces espèces, on a essayé de déterminer : a) son degré de sensibilité à la souche ovestafricaine de T. spiralis,

b) le rôle éventuel qu'elle pourrait jouer accidentellement comme hôte de transmission ou comme réservar de parasites,

c) les possibilités d'adaptation de la souche à un hôte considéré actuellement comme très peu réceptıf, ou partiellement réfractaire.

\section{Chat.}

Considérant les difficultés rencontrées par NELSON et MUKUNDI (1963) pour isoler la souche est-africaine de $T$. spiralis (mauvaise réceptivité du rat et de la souris), nous avons utilisé le chat domestıque pour fixer au laboratoire la souche ovest-africaıne. En effet, $T$. sprralis, essentiellement parasite de carnivores et infestant naturéllement le chacal en Afrique de l'Ouest, le chat, animal d'entretien facile au laboratoire, était tout indiqué pour l'isolement de cette souche. Les résultats ont été excellents. Depuis janvier 1967, nous la conservons sur ce carnivore qui, à notre connaissance, ne sert pas habituellement d'hôte expérimental.

Le contrôle et le calcul du taux d'infestation sont faits, soit par autopsie (moyenne établie d'après les chiffres recueillis à l'examen des muscles de la cuisse, de l'épaule, du dos, du diaphragme et du cou), soit sur biopsie effectuée sous anesthésie générale au niveau de la cuisse, de l'épaule ou des intercostaux. L'importance de chaque prélèvement nécropsique est d'envi. ron 1 gramme, celui de la biopsie 0,2 à $0,5 \mathrm{~g}$.

Le graphique 1 fournit le taux d'infestation musculaire en fonction du nombre de kystes larvaires administrés à l'anımal (nombre de kystes pour 100 grammes de poids vif). 


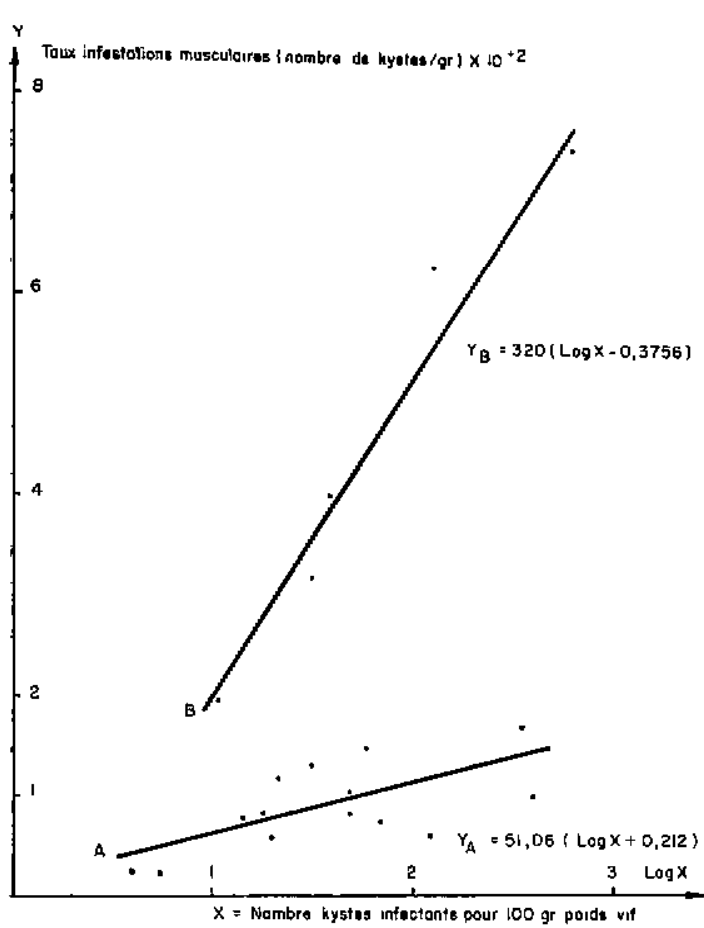

Graphique. - Courbes de sensibilité ef de réceptivilé du chat domestique à la souche ovest-africaine de T. spiralis.

(Les indices de corrélation entre $\log x$ et $y$ pour ces deux droites établies suivant la méthode des moindres carrés sont respectivement :

$$
\begin{aligned}
& \text { courbe } \mathrm{Ar}=0,984 \\
& \text { courbe } \mathrm{Br}=0,695
\end{aligned}
$$

soit une signification supérieure à 99 p. 100.)

Quelques remarques semblent nécessaires en ce qui concerne la sensibilité du chat domestique à la souche ovest-africaıne de T. spiralis.

10 Un très petıt nombre de kystes larvaires (quelques dizaines) suffit à infester convenablement un chat adulte, sans troubles apparents durant la phase intestinale de la maladie.

20 La dose moyenne infestante se situe pour le chat entre 400 ef 600 kysies. Une légère diarrhée apparaît dans les quelques jours qui suivent l'administration de la viande parasitée. Elle disparaît vers les $15^{\mathrm{e}}$ ou $20^{\mathrm{e}}$ jours. La densité du parasitisme trouvé à l'autopsie ou sur biopsie est en général moyenne ( 51 à 121 larves par gramme de muscle).

30 Une augmentation importante du nombre de kystes infestants $(2.000$ à 9.500 kystes pour un chat adulte, correspondant à 125 à 860 kystes pour 100 grammes de poids vif de l'hôte), se traduit par une augmentation du taux d'infestation musculaire (Cf. graphique $n^{0} 1$ ).

\section{Cependant :}

a) Une infestation massive (125 à 860 kystes pour 100 grammes de poids vif), peut être mortelle pour des chats dont l'état général est plus ou morns déficient ( 4 décès sur 23 animaux en expérimentation). Au cours des deux premières semaines apparaît une dıarrhée profuse et hémorragique. L'animal très abattu, fiévreux, est faible, anémié, sans appétit. La mort survient 6 à 20 jours après l'infestation. L'examen des fèces pendant toute la durée des troubles gastro-intestinaux révèle l'existence d'adultes de trichines expulsés avec les excréments lors de la débâcle intestinale.

A l'autopsie, les lésions principales sont une congestion intestinale intense avec entérite hémorragıque, de la congestion hépatique, et un léger hydropéricarde. L'examen du raclat de la muqueuse intestinale met en évidence de nombreux adultes mâles et femelles de T. spiralis.

b) Sur des anımaux en bonne santé, des désordres intestinaux (diarrhée plus ou moins grave avec inappétence passagère) apparaissent dès que le nombre de kystes administrés dépasse 10 pour 100 grammes de poids vif. Les troubles morbides s'aggravent (diarrhée sanglante, vomissements, abatjement et légère hyperthermie) à partır de doses dépassant 50 kystes pour 100 grammes de poids vif.

L'autopsie et la biopsie musculaıre ne révèlent que des infestations de moyenne importance (59 à 172 kystes au gramme de muscle) (graphique 1 . courbe A).

c) Quelques sujets ( 5 sur 19 ), soit environ 26 p. 100 , se sont montrés particulièrement sensibles (graphique 1, courbe B).

Ces animaux ont un taux d'infestation musculaire particulièrement élevé (318 à 745) sans cependant atteindre le millier de larves par gramme de muscle. Ce sont des chats adultes dont la croissance était pratıquement terminée au moment de l'infestation.

C'est peut-être une des raisons pour lesquelles le taux de parasitısme musculaire n'a pas été réduit par une élévation du poids de l'animal.

D'une manière générale, chez tous les animaux ayant eu des troubles intestinaux impor- 
tants, une diarrhée chronique à allure cyclique (8 à 10 jours) persiste pendant plusieurs mols, cependant sans répercussion apparente sur l'état général.

La courbe $A: Y_{A}=51,06(\log . x+0,212)$

(chats jeunes dont la croissance $n$ 'est pas terminée) et

La courbe $B: Y_{B}=320 \quad(\log . x-0,3756)$

(chats adultes ayant terminé leur croissance) démontrent la proportionnalité entre la dose infestante et le taux d'infestation musculaire. C'est, à notre avis, une preuve supplémentaire de l'affinité de la souche ouest-africaine pour les félidés.

Une infestation par 2 d̀ 6.000 kystes peut provoquer l'avortement ou le part prématuré chez la chatte gestante (expérimentation faite sur deux femelles pleines respectivement de 35 et 40 jours).

L'examen trichınoscopique des cadavres de deux chatons nés prématurés, fait 33 jours après leur naissance a été négatif. Les larves de $T$. spiralis ne passeraient donc pas la barrière placentaire chez les félins.

\section{Chien.}

La sensibilité et la réceptivité du chien domestique pour les souches sénégalaises de $T$. spiralis ont été testées sur 6 chiens indigènes de race indéterminée.

Des doses moyennes de 10 à 25 kystes par 100 grammes de poids vif ne provoquent aucun désordre apparent chez le chien. 80 kystes font apparaître de légers troubles intestinaux qui deviennent alarmants (diarrhée hémorragique, abattement, inappétence) chez les sujets infestés avec plus de 125 larves pour 100 grammes de poids vif.

Contrairement à ce qui se passe chez le chat domestique le taux d'infestation musculare 5 à 520 kystes par g ne semble pas proportionnel à la dose infestante, et le taux de réceptivité est très variable d'un animal à l'autre.

\section{Porc.}

Cette espèce, hôte par excellence de $T$. spiralis en Europe et aux U. S. A. dans les régions où la trichinose règne à l'état endémique, et source principale d'infestation de l'Homme, devait être testée au point de vue sensibilité et réceptivité à la souche ovest-africaine.

Les essais ont été faits sur 14 porcs de race Large White âgés de 4 à 10 mois et pesant de 25 à $65 \mathrm{~kg}$.

Ces anımaux ont été infestés à l'aide de viande de chat ou de chien parasitée à raison de 50 à 300 kystes par gramme de muscle.

Le tableau $n^{0} 1$ donne les résultats généraux obtenus au cours de cette expérimentation.

Le porc de race européenne, même soumı à une infestation massive par la souche ouestafricaine de T. sprralis (plus de 500 kystes pour 100 grammes de poids vif) n'est nullement incommodé dans les jours ef les semaines qui survent. L'appétit est conservé, les gains de poids sont normaux et il ne se produit aucun trouble d'ordre général ou intestinal. A l'examen des fèces, pendant les trois semaines suivant l'infestation, on ne trouve que de très rares trichines adultes.

La recherche des larves migratrices dans le sang circulant chez 4 jeunes porcs ne donne que des chıffres très bas $(0,3$ à 1,3 larve par cc. de sang) avec un maximum entre les $20^{\circ}$ ef $25^{\circ}$ jours. Les prélèvements de sang circulant $15 \mathrm{cc}$. en moyenne) sont faits par ponction cardiaque sur l'animal sous anesthésie générale, les $10^{\mathrm{e}}, 1 \mathrm{e}^{\mathrm{e}}$, $22 \mathrm{e}, 33 \mathrm{e}, 45 \mathrm{e}$ et $55 \mathrm{e}$ jours après l'infestation (GRÉTILLAT et VASSILIADĖS, 1968). Pour le dénombrement et la mise en évidence des larves de T. spiralis, Il a été utilisé la technique exposée par NELSON et BLACKIE en 1966 pour la recherche des mêmes éléments dans le sang circulant chez le rat.

Les autopsies faites au bout de 28 à 198 jours ne donnent que des résultats très faibles en ce qui concerne le taux moyen d'infestation musculaire.

Même chez les animaux ayant absorbé un très grand nombre de larves infestantes (40 à 500 kystes par 100 grammes de poids vif équivalent à $6 \times 10^{+3}$ à $25 \times 10^{+4}$ iarves suivant le poids de l'animal), le nombre de kystes pour un gramme de muscle est très faible : 1 à 15 en moyenne pour attendre rarement une vingtaine dans certains groupes musculaires réputés être des sièges de prédilection, tels que les piliers du diaphragme.

Si la densité moyenne oscille autour de 2 à 15 kystes larvaires au gramme de muscle, il est à noter qu'un très grand nombre d'entre eux subis- 
sent un début de calcification ou sont calcifiés dès la fin du premier mois, c'est-à-dire presque immédiatement après leur formation.

Cette destruction par calcification débute par la formation d'une zone d'apparence granuleuse de couleur marron plus ou moins clair qui entoure le kyste puis envahit peu à peu son intérieur (photo no 1). Au début, la larve est transparente et mobile avec des organes internes se détachant très nettement du reste de l'helminthe ef une cuticule mince et lisse. Puis apparait un brunissement de la larve qui devient immobile et se plisse superficiellement comme si elle se rétractait sur elle-même. Une fois calcifié, l'ensemble kyste et larve est un élément allongé, plus ou moins ovalaire ou fusiforme, granuleux, jaune brunâtre et de dimensions variables presque toujours supérieures à $500 \mu$ et pouvant atteindre 800 à $1.000 \mu$ (photo n0 2). A l'intérieur, il est possible de reconnaître, sur les kystes récemment calcifiés, les contours plus ou moins estompés de la paroi kystique avec une larve morte, petit boudin noirâtre spiralé ou parfois fragmenté (photo no 3), qui disparaît totalement dans les vieux kystes calcifiés, seulement reconnaissables à leur forme allongée dont la texture granuleuse et marron est un peu plus foncée au centre qu'à la périphérie.

Cette très faible réceptivité de l'espèce porcine à la souche ouest-africaine de T. spiralis peut s'expliquer de la manière suivante:

Malgré un nombre très élevé de larves infestantes, les adultes mâles ef femelles de trichines sont toujours très rares dans l'intestin grêle des porcs infectés $(0,4$ q et $0,15 \mathrm{o} / 1 \mathrm{~cm}$ de duodénum: 0,7 ㅇ ef $0,35 \mathrm{o} / 1 \mathrm{~cm}$ de jejunum ; 0,05 의 et $0,05 \mathrm{o} / 1 \mathrm{~cm}$ d'iléon) (*). Aucun spécimen adulte n'étant pratiquement expulsé avec les matières fécales dans le courant des trois premières semaines $(0,07$ adulte par jour et par $100 \mathrm{~g}$ de féces), une «barrière intestinale » semble protéger le porc contre les infestations massives en empêchant l'évolution normale de la larve jusqu'au stade adulte.

Les femelles et mâles de trichines qui arrivent à maturité ne donnent naissance qu'à un pefit nombre de larves migratrices qui, pour la plu-

(*) Chiffres établis à l'examen de deux porcs autopsiés les $13^{\mathrm{e}}$ et $15 \mathrm{e}$ jours. part, sont détruites par calcification quelques jours ou quelques semaines après leur enkystement dans les muscles du porc (GRÉTILLAT et VASSILIADËS, 1968).

II semble donc permis d'admettre que le porc domestique de race européenne (Large White) est un très mauvais hôte pour la souche ovestafricaine de $T$. spirolis. II ne faut cependant pas être trop affirmatıf, car l'expérimentation le prouve, certains sujets peuvent héberger des kystes de trichines deux à six mois après leur infestation (Cf. tableau no 1). La chair de ces animaux peut infester le chien ou le chat (voir tableau no 2), donc peut-être l'Homme.

Le rôle joué par les réservoirs de virus et par les hôtes occasionnels susceptibles d'assurer accidentellement l'infestation de porcs élevés en semi-liberté, est donc une question très importonte à élucider, tout d'abord par infestation expérimentale au laboratoire, puis au moyen d'enquêtes épıdémiologiques menées sur le terrain.

Quant aux risques et possibılités d'adaptation des souches ovest-africaines de $T$. spiralis au porc domestique, par possages successifs sur le même hôfe, le taux de parasitisme extrêmement bas obtenu chez les animaux d'expérience rend très malaisé la réalisation en série d'infestations massives.

Les essais de passage alterné entre porcin et carnivores domestiques ne permettent pas de conclure (Cf. tableau no 2). En effet, si le carnivore (chien ou chat) s'infeste normalement avec les quelques kystes non calcifiés trouvés chez des porcs relativement sensibles, il ne nous a pas été possible jusqu'à présent de réaliser des passages de porc à porc; les kystes viables obtenus sur porc étant trop rares pour réaliser des infestations appréciables.

\section{Rat blanc.}

Les essais ont porté sur 10 groupes de deux individus d'âge adulte et de poids moyen, sans distınction de sexe avec des doses infestantes allant de 250 à 10.000 kystes par 100 grammes de poids vif d'hôte.

Un premier essai d'infestation sur quelques rats blancs (4) à l'aide de chair de phacochère parasitée se salde par un échec. Le nombre de kystes administrés étant très réduit (8 à 10), l'expé- 
TABLFAU $N^{\circ}$ I

Porcs

\begin{tabular}{|c|c|c|c|c|c|c|c|c|c|c|c|c|c|c|c|c|c|c|}
\hline \multirow{2}{*}{$N^{\circ}$} & \multicolumn{2}{|c|}{$\begin{array}{l}\text { Infestation } \\
\text { (nombre de kystes) }\end{array}$} & \multicolumn{12}{|c|}{$\begin{array}{c}\text { Contrôles nécropsiques (nombre de kystes vivants } \\
\text { ou calcifiês pour un gramme de muscle) }\end{array}$} & \multicolumn{4}{|c|}{$\begin{array}{l}\text { Duffërcnts types de kystes } \\
\text { pourcentages respectifs }\end{array}$} \\
\hline & Total & $\begin{array}{l}\text { pour } 100 \mathrm{~g} \text { de } \\
\text { poids vif }\end{array}$ & 1 & 2 & 3 & 4 & 5 & 6 & 7 & 8 & 9 & 10 & 11 & 12 & 13 & 14 & 15 & 16 \\
\hline 1787 & 60.000 & 513 & 40 & 8 & 34 & 15 & 22 & 11 & 6 & 7 & 8 & 12 & 12 & 4 & & 5 & 60 & 35 \\
\hline 1786 & 60.000 & 500 & 31 & 5 & 4 & 7 & 3 & 1 & 1 & 0 & 7 & 6 & 2 & 4 & 2,5 & & 12,5 & 85 \\
\hline 1785 & 60.000 & 500 & 79 & 2 & 3 & 2 & 2 & 0 & 1 & 0 & 2 & I & 0 & 5 & & & & 100 \\
\hline 1374 & 250.000 & 430 & 56 & 16 & 16 & 6 & 13 & 6 & 2 & 4 & 7 & 18 & & 6 & & 50 & & 50 \\
\hline 608 & 80.000 & 278 & 46 & 3 & 6 & 1 & & 1 & & & 1 & & & 1 & & 30 & & 70 \\
\hline 610 & 100.000 & 238 & 49 & 5 & 11 & 5 & 4 & 6 & 2 & 1 & 3 & 1 & 2 & 1 & & & & 100 \\
\hline 1373 & 100.000 & 232 & 47 & 0 & 1 & 8 & 2 & 0 & 0 & 0 & 5 & 0 & & 2 & & 20 & 30 & 50 \\
\hline 1794 & 32.700 & 182 & 28 & 1 & 2 & 1 & & 3 & & & & 4 & & & & 10 & 10 & 80 \\
\hline 1791 & & 182 & & & & & & & & & & & & & & & & \\
\hline 1792 & & 182 & & & & & & & & & & & & & & & & \\
\hline 1784 & 11.500 & 49 & 62 & 0 & 6 & 0 & 0 & 1 & 0 & 0 & 0 & 0 & o & & & & & 100 \\
\hline 1782 & 14.000 & 48 & 198 & 4 & 4 & 5 & 1 & 1 & 3 & 3 & 4 & 3 & 2 & 2 & & 60 & & 40 \\
\hline 609 & 10.000 & 43 & 57 & 9 & 10 & 4 & & 7 & & & 21 & & & & & & & 100 \\
\hline 1783 & 18.000 & 41 & 111 & 1 & 0 & 2 & 2 & 0 & 1 & 0 & 1. & 1 & & 0 & & & & 100 \\
\hline
\end{tabular}

(1) nombre de jours écoulés entrc l'infestation expérımentale et le contrôle nécropsique. (2) épaule. (3) cuisse. (4) diaphragme,

(5) piliers du diaphragme. (6) masseters. (7) larynx. (8) langue. (9) cou. (10) intercostaux. (11) dos. (12) sous-cutané

(13) larves libres non enkystées. (14) kystes normaux. (15) kystes en voie de calcification, (16) kystes calcifiês. 
TABLEAU $\mathrm{N}^{0}$ II

\begin{tabular}{|c|c|c|c|c|c|c|c|c|c|c|}
\hline \multirow[b]{2}{*}{ Animaux } & \multicolumn{3}{|c|}{ Infestation } & \multicolumn{7}{|c|}{ Contrôle nēcropsique (kystes/g muscle) } \\
\hline & par & $\begin{array}{c}\text { Nombre } \\
\text { kystes } / 100 \mathrm{~g}\end{array}$ & $\begin{array}{l}\text { Nombre total } \\
\text { de kystes }\end{array}$ & $\begin{array}{c}\text { après } \\
\text { (nombre jours) }\end{array}$ & Epaule & Cuisse & Diaphragme & Masseter & Cou & $\begin{array}{l}\text { Taux de kystes } \\
\text { calcifiés }\end{array}$ \\
\hline lorc $n=1374$ & Chien & 430 & $25 \times 10^{+4}$ & 56 & 16 & 16 & 6 & 6 & 7 & + ou -50 p. 100 \\
\hline Chat $n^{\bullet} 33$ & Porc n' 1374 & 200 & $32 \times 10^{+2}$ & 62 & 112 & 71 & 60 & 63 & 70 & 0 p.100 \\
\hline Forc $n^{\circ} 608$ & Chat $n^{\circ} 33$ & 278 & $8 \times 10^{+4}$ & 46 & 3 & 6 & 1 & 1 & 1 & 70 p. 100 \\
\hline Forc $n^{0} 1343$ & Chien & 232 & $10+5$ & 47 & 0 & 1 & 8 & 0 & 5 & + ou $-80 \mathrm{p} .100$ \\
\hline Chien $n^{\circ} 1 \mathrm{l}$ & Porc $n^{\circ} 1373$ & 13 & 640 & 60 & 27 & 24 & 48 & 37 & 21 & $0 \mathrm{p} .200$ \\
\hline Porc $n^{\circ} 610$ & Chien $n^{0} 11$ & 238 & $10^{+5}$ & 49 & 5 & 11 & 5 & 6 & 3 & $100 \mathrm{p.j} 00$ \\
\hline Porc $n^{\circ} 1782$ & Chat & 48 & $14 \times 10^{+3}$ & 198 & 4 & 4 & 5 & 1 & 4 & + ou -40 p. 100 \\
\hline Chat $n^{\circ} 42$ & Porc $n \cdot 1782$ & 5 & 150 & 66 & 12 & 10 & 8 & 0 & 2 & $0 \mathrm{p} .100$ \\
\hline Pore $n^{\circ} 410$ & Chat $n^{0} 42$ & 81 & $12,6 \times 10^{+3}$ & 26 & 8 & 1 & 0 & 1 & 8 & $90 \mathrm{p} .100$ \\
\hline
\end{tabular}






Photos 1 à 3. - Kystes calcifiés chez le porc. 
rimentation se poursult en utilisant des doses infestantes plus fortes, de l'ordre de quelques centaines, voire de plusieurs milliers de kysies pour 100 grammes de poids vif d'hôte.

Le rat blanc est très peu sensible à la souche ovest-africaine de $T$. spiralis. Il est nécessaire de faire avaler 1.000 à 1.500 kystes à un rat blanc adulte pour obtenir un très faible parasitisme musculaire : 6 à 37 kystes ou larves libres par gramme de muscle, avec un très fort pourcentage (50 à 100 p. 100) de kystes calcifiés ou en voie de calcification (Cf. photos $n^{0 s} 4,5,6$ ef 7).

Tout comme chez le porc domestique, le cycle biologique semble enrayé en deux points :

10 Une grande partie des larves infestantes sont détruites au niveau de l'intestin et ne se transforment pas en adultes puisque chez les animaux autopsiés dans les 3 à 4 semaines suivant l'infestation, on ne retrouve d'adultes de trichine que chez ceux ayant avalé plus de 1.000 kystes par 100 grammes de poids vif d'hôte.

$2^{\circ}$ Dans les cas où les doses infestantes varient entre 1.000 et 1.500 kystes, les quelques adultes présents dans l'intestin du rat, lâchent dans le sang des larves migratrices dont l'enkystement est difficile (larves libres trouvées dans les muscles) ou qui meurent et sont très tôt calcifiées dès qu'elles sont enkystées.

$3^{\circ}$ Au-dessus de 3.000 kystes par animal, le rat blanc meurt au bout de 5 à 20 jours, soit au début (phase intestinale), soit dès l'envahıssement des muscles par les larves. L'autopsie révèle alors de nombreux adulìes mâles et femelles dans l'intestın grêle.

\section{Rat de gambie (Cricetomys gambianus).}

Ce rongeur, très fréquent au Sénégal, affectionne particulièrement les agglomérations humaines, villes et villages, ou il trouve facilement sa nourriture. Omnivore et volontiers carnivore, on le trouve en ville dans les magasins, entrepôts, les jardins, et à la campagne, aux alentours des villages où Il se nourrit de tubercules (patates douces), de graines de plantes vivrières (maïs, mil, sorgho...), et de détritus divers.

En contact permanent avec le porc domestique dont la seule alimentation est souvent celle qu'il trouve dans les champs et les ruelles du village, i] pourrait éventuellement jover le rôle, sinon de réservoir de parasites, du moins celui d'hôte de passage.

Une série de tests a été réalisée sur $17 \mathrm{C}$. gambianus adultes pesant environ $0,7 \mathrm{~kg}(0,5$ d̀ $0,95 \mathrm{~kg}$ ) et capturés dans un entrepôt de la ville de Dakar.

Un premier lot de cinq individus est infesté avec des doses allant de 100 à 1.800 kystes par sujet (24 à 145 kystes par 100 grammes de poids vif d'hôte).

Parasités massivement par des cestodes, des nématodes et des coccidies, ces rongeurs meurent 8 à 32 jours après leur infestation. A l'autopsie, de très nombreux adultes de $T$. spiralis mâles et femelles sont présents dans l'intestın grêle. Chez l'un d'entre eux, mort au bout de 32 jours, quelques larves non enkystées sont retrouvées entre les fibres musculaires.

Les essais sont poursuivis sur des animaux préalablement vermifugés pour les débarrasser de leurs parasites gastro-infestınaux et les rendre moins vulnérables à l'action pathogène de T. spiralis.

Contre les coccidies du 1-(4-amino-2-n-propyl(-pyrimidınylmethyl)-2-picolonium chloride hydrochloride (Amprolium) est ajouté pendant 6 jours dans la ration (50 mg/kg/jour).

Les cestodes intestinaux sont détruits en donnant par voie orale, $3 \mathrm{mg}$ par animal deux fois à 3 jours d'intervalle de l'Acide-3-acetylamino-4-hydroxy-phenylarsonate d'arécoline.

Quant aux nématodes, le 0.1. tetrahydro-e,3, (,-,phenyl-amido (2,1-b) thiazole chlorhydrate, deux traitements à 3 jours d'ıntervalle à raison de $7,5 \mathrm{mg} /$ par animal, suffit à les détruire (Tétramizole).

Des examens coprologiques faits une semaine après cette sérıe de traitements confirment l'efficacité de la cure, l'expérimentation pouvant débuter sur des animaux pratiquement débarrassés de leurs parasites gastro-intestinaux.

6 lots de 1 à 3 Cricetomys (12 animaux) infestés avec des doses allant de 100 à 600 kystes par animal sont autopsiés pour contrôle 49 à 80 jours après l'infestation. A part un seul sujet mort accidentellement au bout de 8 jours, tous les autres ont pu être suivis et contrôlés efficacement.

Interprétation des résultats :

Le rat de Gambie est réceptif à la souche ovestafricaine de T. spiralis puisque 100 d̀ 200 kystes 


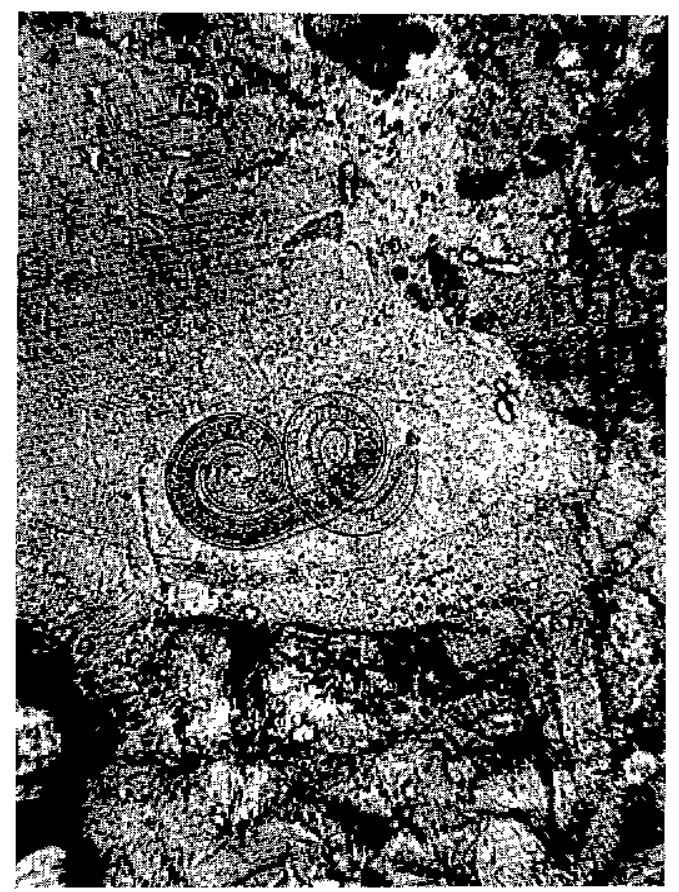

Photo 4. - Larve vivante placée dans un kyste aux parois indistinctes ef très minces (muscle de rat).

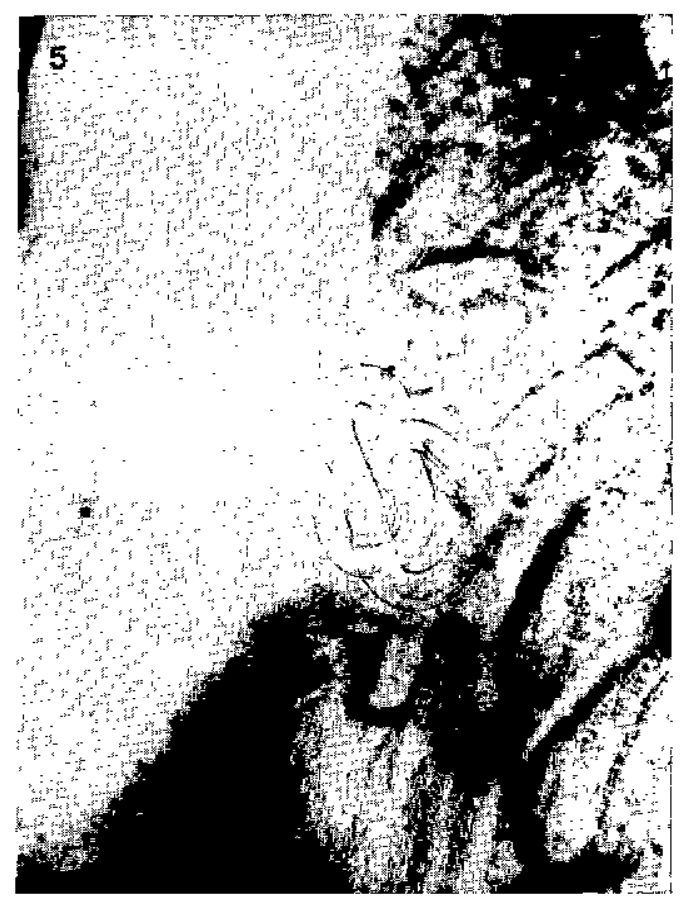

Photo 5. - Larve lıbré dans le muscle du rat.
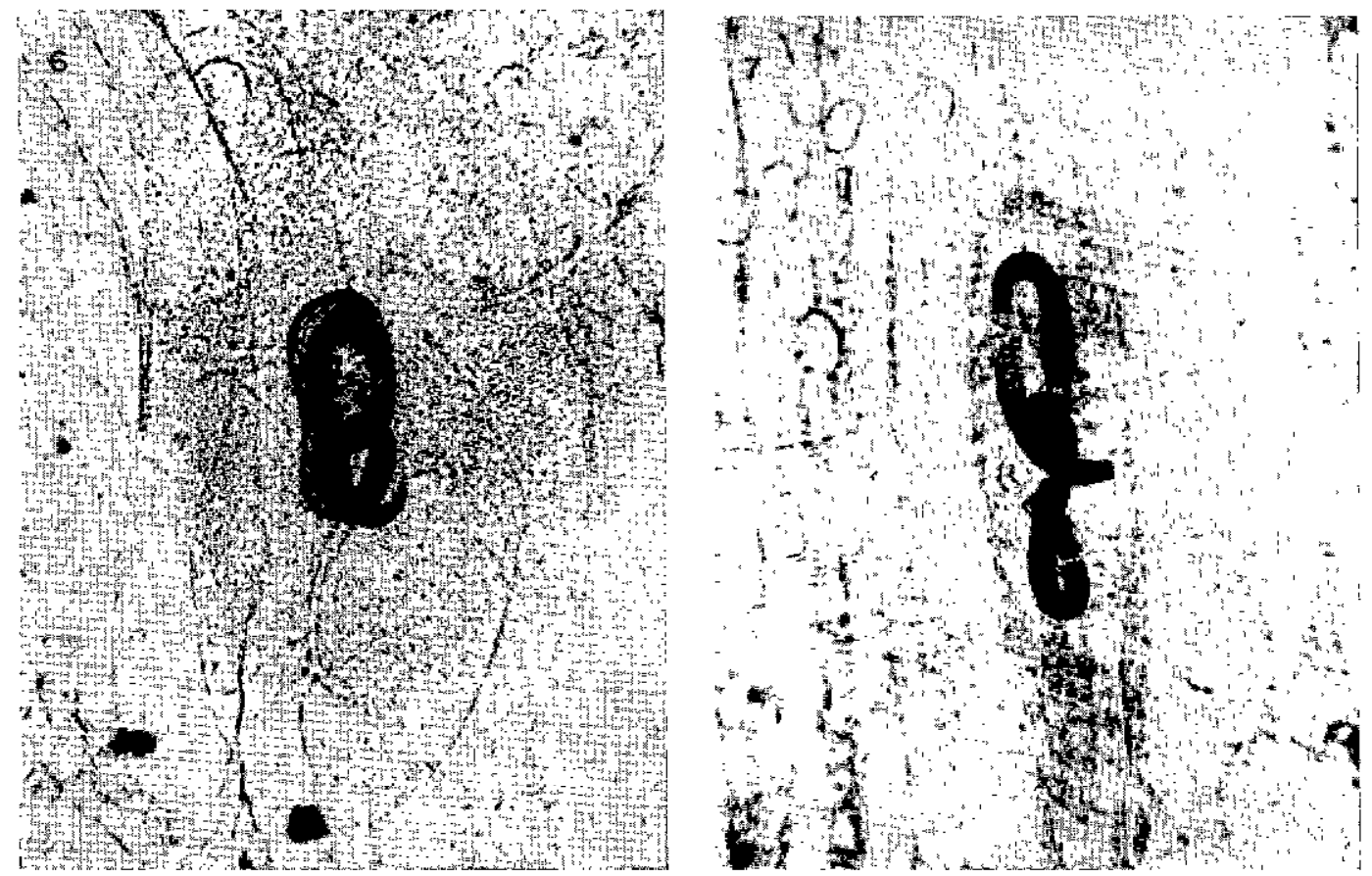

Photos 6 et 7. - Larves et kyste calcifiés chez le rat. 
par animal, soit sensiblement 10 à 17 kystes par 100 grammes de poids vif d'hôte, suffisent à infester moyennement un Cricetomys. Un pourcentage cependant appréciable de kystes sont calcifiés à partir du $50^{\mathrm{e}}$ jour après l'infestation, ce qui laisse supposer une destruction complète des kystes au bout de 3 à 5 mois.

D'autre part, le polyparasitisme massif dont sont atteints la plupart de ces rongeurs, les rend très vulnérables à l'action pathogène de $T$. spiralis puisque même une faible infestation suffit à tuer l'animal en quelques jours ou quelques semaines avant l'enkystement des formes larvaires dans les muscles.

De tout ceci, faut-il conclure de l'impossibilité qu'a ce rongeur de jouer un rôle dans les risques de transmıssion de la trichınose au porc domestique. Si l'on considere la fréquence de ce mammifère et ses nombreux contacts avec la faune sauvage, il pourrait représenter dans certains cas un chaînon dans le cycle de la trichine comme hôte de passage éventuel, mals sporadique. II peut difficilement être consıdéré comme un réservoir de parasites.

\section{Souris.}

La souris blanche, hôte de choix pour les souches européennes de $T$. spiralis, devait être testée au point de vue réceptivité et sensibilité à la souche ovest-africaine.

Les premiers essais d'infestation réalisés avec quelques kystes par souris se soldèrent par un échec (GRÉTILLAT et VASSILIADĖS, 1967).

Ils sont repris en utilisant six lots de 5 souris chacun, d'un poids moyen de $22,50 \mathrm{~g}, 3$ lots de femelles et 3 lots de mâles, infestés respectıvement avec 25, 50 et 100 kystes larvaires. Un lot de 5 souris est conservé comme témoin.

L'admınistration des kystes est fatte à la pınce, souris par souris, à l'alde de menus fragments de chair de chat parasité à raison de 500 larves par gramme de muscle.

Aucune mortalité n'ayant été relevée, tant chez les témoins que chez les infestés, 45 jours après le début de l'expérimentation, les souris sont autopsiées pour contrôle.

Pour établir une bonne moyenne du taux d'infestation musculaire, sont examinés en plus du diaphragme, des fragments de masseter, d'épaule, de cuisse, des intercostaux et des muscles abdominaux, l'ensemble pesant environ $0,600 \mathrm{~g}$. Pour un contrôle plus exact et pour éviter les erreurs possibles lors de l'extrapolation des résultats abtenus sur un poids de chair aussi faible, l'ensemble des muscles des deux souris les plus parasitées de chaque lot' est examiné pour établır un décompte exact de toutes les formes larvaires présentes dans la carcasse.

Les seuls muscles parasités sont le diaphragme, les masseters ef les peauciers abdominaux. Parmi les kystes larvaires trouvés, 30 à 40 p. 100 d'entre eux sont calcifiés ou en voie de calcification. 40 p. 100 des larves sont libres et non encore enkystées, le reste se présentant sous forme de kystes plus ou moins allongés à contours Indistincts, mais avec cependant une larve bien vivante à leur intérieur.

En résumé, les deux souris (sur 30 en expérimentation) qui se sont révélées les plus réceptives ont fournı respectivement 31 et 53 kystes larvaires dans les 6 à 7 grammes de chair de leur carcasse, alors que la dose infestante était de 100 kystes. Un tel résultat montre combien la souris blanche peut être un mauvais hôte pour la souche ovest-africaine de $T$. spirolis.

\section{Lapin.}

Un premier passage à partır de muscle de chat est fait sur 5 lapins en utilisant des doses allant de 500 à 12.000 kystes par animal. Les taux de parasitisme musculaire obtenus son corrects mais inconstanis. Tous les kystes sont viables. Une dose de plus de 10.000 kystes est mortelle pour le lapin, mass 500 kystes suffisent à obtenir une infestation moyenne.

A partir d'un lapın infesté à raison de 300/1/g, un passage est fait sur 12 lapıns avec des doses individuelles de 500 kystes.

II se produit un amoindrissement de la souche (diminution du taux de parasitisme musculaire et une calcification précoce des kystes : 1 à 18 l/g, avec 28 à 94 p. 100 de kystes calcifiés chez certains sujets).

Il est difficile de considérer le lapın comme un animal utilısable au laboratoire pour conserver ef multiplier la souche ouest-africaine de T. spiralis. 


\section{Hérisson (Atelerix albiventris).}

Cet insectivore est très fréquent au Sénégal. Son régime alimentare très varié va de la proie vivante (faune entomologique hypogée ef épigée) à la chair putréfiée de carcasses d'animaux sauvages morts dans la brousse.

Susceptible de représenter en conséquence un chaînon dans l'épidémiologie de la trichinose en Afrique de l'Ovest, une expérimentation a été réalisée au laboratoire sur 9 spécımens adultes capturés dans la région de Saınt-Lovis du Sénégal.

Atelerıx albiventris peut difficilement être considéré comme un hôte de passage possible ou probable pour la souche ovest-africaine de T. spiralis.

En effet, si des doses de 2 à 400 kystes infestent un hérisson adulte, la phase d'enkystement au niveau du muscle n'a pas lieu ou se prodult de manière imparfalte. Ces larves demeurent libres entre les fibres musculaires. D'autre part, une infestation massive (plus de 600 kystes) est mortelle pour $A$. albiventris qui meurt au moment de l'envahissement de l'organisme par les larves migratrices.

\section{Singe.}

Une expérimentation a été tentée sur singe pour évaluer le pouvoir pathogène de la souche vis-à-vis des Primates.

Un Erythrocebus patas (singe pleureur), âgé environ de 2 ans et 2 Papio papio (cynocéphale) âgés respectivement de 1 an et 2 ans sont infestés avec des doses allant de 600 à 4.500 kystes por animal.

Le singe est extrêmement sensible à cette souche. Après une phase intestınale qui dure une à deux scmaincs ct où prédomine la diarrhée sans que l'appétit et l'état général soient diminués, apparaît vers les 35 d $40^{\circ}$ jours une période dépressive avec perte d'appétit, faiblesse et myalgies. L'animal se déplace difficilement, les membres postérieurs repliés sous lui-même. A des périodes de troubles alarmants où l'animal en hypothermie $\left(35^{\circ} \mathrm{C}\right)$ est dans un état subcomateux, succèdent des moments de mieuxêtre où l'animal cherche à s'alimenter. Chez les deux Papio apparaît de l'œedème de la face et du bassin quelques jours avant la mort survenue les 40 et $44 \mathrm{e}$ jours au moment de la phase d'enkystement des larves dans les muscles.
Al'autopsie, ces deux cynocéphales très amaigris ont un œedème sous-cutané généralisé à toutle corps. Le cadavre ruisselle et dans le liquide d'œdème pullulent des larves de trichine non encore enkystées. Congestion intestinale et hépatique. A l'examen trichinoscopique, le taux d'infestation musculaire est très élevé (707 et $270 \mathrm{l} / \mathrm{g}$ en moyenne) mais la presque totalité des larves sont libres (85 à 95 p. 100). L'animal est mort au moment de l'enkystement des larves.

Quant à l'E. patas, après avoir présenté des troubles alarmants vers le 40 jour, abattement, décubitus latéral sans possibilité de se déplacer, mass avec un appétit conservé, son état s'améliore vers le 50 jour. Le $66 \mathrm{e}$ jour se prodult une rechute. Efat comateux, hypothermie, la mort survient 24 heures après, malgré un traltement aux corticoides.

Le cadavre très amaigri n'est pas cedématié et aucune lésion particulière n'est à signaler au niveau des viscères. L'examen trichinoscopique fournit un taux d'infestation musculare moyen de 1.080 kystes par gramme de muscle. L'animal est mort après la fin de la phase d'enkystement des larves.

\section{Phacochère (Phacochoerus aethiopicus).}

La souche conservée et entretenue au laboratoire ayant été isolée à partir de ce mammifère, qui est à l'origine d'un certain nombre de cas de trachınose humaıne au Sénégal, nous pensans que les résultats des enquêtes sur la fréquence et le taux de parasitisme par $T$. sprralis de ce suidé trouvent leur place dans cette étude, sans parler de leur import ance au point de vue parasıtologique, épidémılologique et sanitaire.

Au cours de la saison de chasse 1966/67, la chair (en particulier les cuisses et les épaules) de 196 phacochères jeunes et adultes abattus dans différentes régions du Sénégal, fut examinée. Le tableau $n^{0} 3$ donne les résultats de cette enquête.

$\mathrm{Si}$ on compare les taux d'infestation obtenus chez le porc infesté expérımentalement avec ceux trouvés chez le phacochère, on peut estimer que ces deux espèces sont très proches au point de vue réceptivité et sensibilıté et qu'en définitive ce sont des hôtes très médiocres pour la souche ovest-africasne de T. spiralis. 
TABLEAU $\mathrm{N}^{\bullet}$ III

(Phacochoerus aethiopicus)

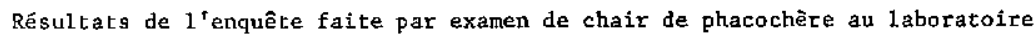

\begin{tabular}{|c|c|c|c|c|}
\hline origine & $\begin{array}{c}\text { Nombre } \\
\text { d'animaux } \\
\text { examinés }\end{array}$ & négatifs & positifs & Organes parasitês \\
\hline De1ta du Sênēgal & 145 & 126 & 9 & $\begin{array}{l}\text { jambor }(0,3)(16)(14)(15)(59)(1) \\
(0,5)(0,5) \text { - masseter }(7,5)\end{array}$ \\
\hline Lac de Guiers & 22 & 22 & 1 & f ambon $(0,35)$ \\
\hline Dakar & 6 & 6 & & \\
\hline Sine Saloum & 2 & 2 & & \\
\hline Inđêt terminēe & 20 & 20 & & \\
\hline Mauritanie & 1 & 1 & & \\
\hline Totaux & 196 & 176 & 10 & \\
\hline Pourcentage & $100 \mathrm{p} \cdot 100$ & $89 \mathrm{p}, 100$ & 11 p. 100 & \\
\hline
\end{tabular}

Légende : $(0,35)$ nombre de larves ou de kystes par grame de muscle examiné..

Remarque : Parmi les 11 p.100 d'animaux parasités, 2 phacochères n'étaient porteurs que de très rares kystes calcifiés, 3 étaient très faiblement parasités (1 ne présentait dans son jambon qu'une larve zu granme de chair). Le pourcentage réel d'animaux vraiment parasitếs capables de transmettre la trichinose d'une mantère certaine est donc de 6,5 p.100 envífon avec un taux d'infestation allant de 15 à 59 larves enkystếs par gramne de muscle.

Lo consommation de chair de phacochère en provenance de la région du Delta du Fleuve Sénégal, sous forme de jambon cru salé ou fumé représente néanmoins un danger certain, 6 à 10 p. 100 de cette viande devant être consıdérée comme trichınée.

Au sujet de l'épidémiologie de la trichine des animaux sauvages dans cette partie de I'Afrique de l'Ouest, il est un peu trop tôt pour conclure.

\section{DISCUSSION ET CONCLUSION}

N'infectant que très difficilement et très légèrement le porc, le rat et la souris, la souche ouestafricaine de $T$. spiralis est très proche de celle isolée par FORESTER et Coll. au Kenya en 1961. Les taux d'infestation obtenus sur souris, rat, lapin par NELSON et Coll. en 1966, sont cependant beaucoup pius hauts que ceux que nous obtenons. Un travail est en cours sur cobaye pour voir dans quelle mesure ce rongeur peut servir à la conservation et à la multıplication de la souche.

Essentiellement adaptée aux carnivores et particulièrement aux Félidés, la trichinose africaine n'affecte pas les Muridés. Elle est en cela complètement différente des trichinoses euro- péenne et américaine où l'infestation de l'Homme a lieu à partir du porc.

Si le taux d'infestation du phacochère en Afrique de l'Ouest semble assez faible, les risques de contamination par consommation de viande de chasse mal cuite ou crue (jambon de Bayonne) sont réels. Au sujet du danger de voir le porc d'élevage contracter la trichınose en avalant de la chair d'animal parasité, les élevages porcins sont en général très éloignés des terrains de parcours du phacochère ef le taux d'infestation de ce dernier permet difficilement à un porc adulte de s'ınfester d'une manière appréciable. D'autre part, les Muridés comme nous venons de le volr peuvent difficilement servir de chaînon intermédiaire pour transmettre la maladie.

II y a lieu cependant d'être vigilant à ce sujet car le porc domestique presque aussi réceptif que le phacochère, peut être infesté accıdentellement par une souche qui, si elle est moins ubiquiste que ses homologues européenne et américaine, n'en est pas moins extrêmement pathogène pour l'Homme.

Institut d'Elevage et de Médecine vétérinaire des Poys tropicoux - Maisons-Alfort.

Laboratoire notional de l'Elevage

ef de recherches vétérinoires - Dokar-Hann. 


\title{
SUMMARY
}

\author{
Biological particularities of the West African strain \\ of Trichinella spiralis (Owen 1835). Receptivity \\ and susceptibility of some domestic and wild mammals
}

Several tests of receptivity and susceptibility have been carried out on 23 cals, 6 dogs, 14 pigs, 22 rats, 30 mice, 17 Gambian Giant Rat (Cricetomys gambianus), 17 rabbits, 9 hedgehogs (Atelerix albiventris) and 3 monkeys, which have been infested experimentally with the West African strain of Trichinella spiralis.

Following results have been recorded:

a) The domestic cat is very receptive and susceptible, but the strain is moderatly pathogenic for it. The clinical signs consist in hemorragic dıarrheae which occur chronically in the cases of massive infestation.

The rate of muscles infestation by the encysted larvae is pratically proportionnal to the infestive dose which has been given (number of infestive cysts ingested per $100 \mathrm{~g}$ of living weight of the host).

b) The dog is less often infested than the cat, but is still very susceptible. Results found in post-mortem examinations are not proportionnal to the infestive doses.

c) The domestic pig (European breed «Large Whote ») has a very low susceptibility. Most of the infestive larvae are destroyed by the « intestınal barrier », before they develop their adult form, and the few migrating ones which are reaching the muscles undergo a process of calcification as soon as they get encysted, or in the following days or weeks.

However in some animals, some cysts survive and remain infestive during several months. This does not exclude the danger of outbreak of trichinosis caused by successive passages on pig.

d) The wild rodents : rat, mouse and Gambian rat are very bad hosts, for various reasons : they are refractory, the larvae give abnormal cyst or not at all, calcification of the cyst and of the larvae accompagnied by sterilization of the body a few weeks after infestation. Therefore, the wild rodents can hardly play a role in the epidemiology and the spread of trichinosis in West Africa.

e) The local hedgehog (Atelerix albiventris) has no role in this epidemiology.

f) The rabbit, as laboratory experimental host, reacts very badly against this strain and its receptivity is to uncertain and variable to use it for the conservation of the straın.

g) The primates (monkeys Papio papio and Erythrocebus potos) are very susceptıble. A dose of about hundred cystsis lethal for a young baboon. Carnivora and particularly the felidae are the elective hosts of the trichine in West Africa. The wild carnivora are reservoirs of parasites; the warthog is only an accasional host ( 6 to $10 \mathrm{p}, 100$ of the killed animals) and, like the domestic pig, get infested with difficulty.

\section{RESUMEN}

Particularidades bialógicas de la cepa ceste-africana de' Trichinella spirolis (Owen, 1835). Receptividad y sensibilidad de algunos mamíferos doméstıcos y salvajes

Se efectuaron varias series de pruebas de receptividad y de sensibilidad en 23 gatos, 6 perros, 14 cerdos, 22 ratas, 30 ratones, 17 ratas de Gambia (Cricetomys gambianus), 17 conejos, 9 erizos (Atelerix abiventris) y 3 monos, experimentalmente infectados con la cepa oesteafricana de Trichinelia spiralis.

Se notan los resultados siguientes:

a) El gato doméstico es muy receptivo y susceptible pero la cepa es medianaumente palógena para él. Se limitan los desórdenes mórbidos a una dıarrea 
hemorrágica que va haciendo crónica en los casos de infestación maciza. La tasa del parasitısmo muscular mediante larvas enquistadas es casi proporcional a la dosis infestante administrada (número de quistes infestantes ingeridos por 100 gramos de peso vivo del huesped).

b) El perro es menos infestado que el gato, sin embargo es muy susceptible. No son proporcionaies a las dosis infestantes los resultados encontrados durante las autopsias de control.

c) En cuanto al cerdo doméstico (raza europea Large White), es poco susceptible. Una barrera intestinal destruye la casi totalidad de las larvas infestantes antes que sean adultas y las larvas migradoras llegando al nivel de los musculos van caicificandose desde su enquistomiento o durante los dias o semanas sıguientes. Sin embargo, en un clerto número de casos, algunos quistes sobreviven y quedan infestantes durante varios meses, lo que no exclue el riesga de ocurrencia de casos de triquinosis de los cerdos par pasajes sucesivos en un mismo huesped.

d) Los roedores salvajes : la rata, el ratón y la rata de Gambia son huespedes muy malas par que son refractarios ; las larvas se enquistan anormalmente o no; se notan una calcificación de los quistes y de las larvas y la esterilización del organismo algunas semanas después de la infestación. Por consecuencia, los roedores salvajes pueden dificılmente desempeñar un papel en la epidemiologia y la dispersión de la triquinosis en Africa del Oeste.

e) No desempeña nıngún papel el erızo local (Atelerix abiviventris) en dicha epidemiologic.

f) El conejo, como huesped experimental de laboratorio, reacciona muy mal para con esta cepa; no se puede utilizarlo para la conservación de la cepa siendo su receptividad demasıado incierta y variable.

g) Los primates (monos Papio papio y Erythrocebus patos) son muy susceptibles. Una dosis de unas centenas de quistes es mortal para un joven cinocefalo. Los carnivoros y particularmente los felidos son los huespedes de elección para la triquinosıs en Africa del Oeste, los carnivoros salvajes son reservorıos de parásitos ; el facoquerio no es más que un huesped ocasional ( 6 a 10 p. 100 de los animales matados examinados) infestandose dificilmente ası como el cerdo doméstico.

\section{BIBLIOGRAPHIE}

FORESTER (A. T. T.), NELSON (G. S.) et SANDER (G.). - The first record of an outbreak of trichinosis in Africa south of the Sahara. Trans. Roy. Soc. frop. Med. Hyg., 1961, LV, 503.

GRETILLAT (S.). et VASSILIADES (G.). - Présence de Trichinella spiralis (Owen, 1835) chez les carnivores ef suidés sauvages de la région du Delta du Fleuve Sénégal. C.R. Acod, Sci, Paris, 1967, CCLXIV : 1297-1300.

GRETILLAT (S.) et VASSILIADES (G.) - Réceptlvités comparées du chat et du porc domestique à la souche ouest-africaine de Trichinella spiralis (Owen, 1835). C. R. Acad. Sct. Paris, 1968. A paraître.
KOZAR (Z.) et KOZAR (N.). - A comparison of the infectivity and pathogenicity of Trichinella spiralis strains from Poland and Kenya. I. Helminit., 1965, XXXIX (1) : 19-34.

NELSON (G.S.) et BLACKIE (E. J.). - Comparative studies of geographical strains of Trichinella spiralis. Trans. Roy. Soc. trop. Med. Hyg., 1966, LX (4) : 471-480.

NELSON (G. S.) et MUKUNDI (J.). - A strain of Trichinella spiralis from Kenya of low infectivity to rats and domestic pigs. J. Helminth., 1963, XXXVII (4) : 329338. 